\title{
Application of CAD/CFD/RP-technologies for development of LVAD systems
}

\author{
Valentin Morozov, Alexey Zhdanov and Leonid Belyaev \\ Department of Mechanical Engineering, Vladimir State University named after Alexander and Nikolay Stoletovs, Vladimir, Russia
}

\begin{abstract}
The important problem of new Left Ventricle Assist Device (LVAD) systems development is to determine their hemodynamic and functional characteristics and reduce the design time. An effective method for achieving this is the use of approaches based on the application of CAD/CFD/PR-technologies. Methods of computer modelling were applied to determine the geometrical parameters of the blood pump, and methods of finite element analysis were used for the optimization of hemodynamic parameters of the LVAD system. The geometrical modelling was carried out in the Pro/Engineer WF5. The CAE-system Ansys was used to analyze the blood flow in the chamber of the pump. The motion of the flexible walls was simulated by normal flow through rigid walls. At the final stage some models were prepared for prototyping. The critical parameters of hemodynamic gave the possibility to optimize the shape of the blood pump. The LVAD system and its components were made using various RP-technologies. The hydraulic tests for the performance investigation and the analysis of implantability for implantable systems were carried out for manufactured prototypes of LVAD systems. The manufactured components of the LVAD system are completely operability and give the possibility to estimate the critical hemodynamic and functional parameters of the LVAD system.
\end{abstract}

\section{Introduction}

During the design of LVAD systems, complex scientific and engineering problems associated with the construction, simulation and experimental researches of the developed systems are solved. The solution of these problems implies the rise in the cost of the design and increases the time of manufacture of the finished product, based only on the comparative experimental studies of different design versions by a successive approaching of the functional characteristics of the systems to the required parameters that are set in the medical and technical requirements. In this regard, the direction associated with the use of the design approach is promising, giving the possibility of finding the optimal geometric design and the hemodynamic parameters of the LVAD systems already in the designing phase of the product. This approach based on the mathematical modelling of the processes realized during the operation of the LVAD systems allows to optimize the design, to compare the alternatives of the developed systems based on the analysis of the results of computational simulations and bed testing of the samples of LVAD systems fully or partially manufactured using rapid prototyping technologies. In this article it is examined the design of pulsative LVAD systems based on the application of $\mathrm{CAD} / \mathrm{CFD} / \mathrm{RP}$-technologies which allow to determine the hemodynamic parameters of the system operation at the design stage and reduce the time of production of the finished article.

\section{Methods}

The creation process of the basic components of LVAD systems is a unified interconnected cycle stages. At all stages of the produced intensive exchange of different kinds of information. An important feature of this information is its bidirectional and multi-step iterative refinement based on the nature of the solutions to global and local problems of analysis, synthesis, optimization and compromise. One of the first steps is the creation of three-dimensional geometric model, which is the basis for all the following stages of designing and creating the LVAD systems. Three-dimensional modelling design LVAD systems is implemented in the Pro/ENGINEER WF5 (PTC, Inc, USA). Obtained at this step data has been transferred to the CAE-system for the hemodynamic analysis. The flow simulation inside the blood chamber was led based on actual geometry of the blood chamber (Fig. 1). The computational model represents the internal volume of the blood chamber based on the actual geometry of the main body, valves, radii and transitions. The simulation with one mitral valve position and various operation modes is described in the preliminary work [1].

This work presents the results of the simulation that was performed in order to determine the optimal position of the mechanical tilting disc valve "MIKS" (LLC "Roskardioinvest", Russia) [2]. This valve have a minimal external diameter $-17 \mathrm{~mm}$ and a geometric hole area of $1.54 \mathrm{~cm}^{2}$. The starting point of the mitral valve 
was chosen as follows: the disk valve was oriented in such a way that its major orifice open to the external side of the inner wall, but the strut keeps perpendicular to the diaphragm. The valve rotation was realized clockwise. The modelling was carried out for the valve positions in 0 , 30 and 45 degrees. The aortic valve position did not change (Fig. 2).
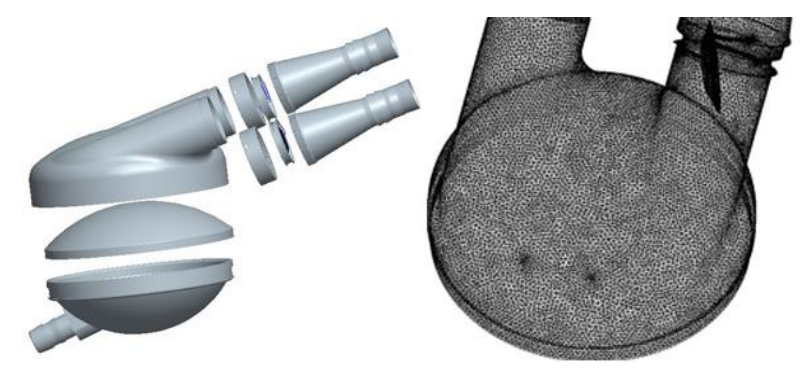

Figure 1. Pneumatic LVAD system: 3D model (left) and the mesh of finite-element model for hemodynamic analysis (right). A concentrate mesh was implemented in the valves zones and in the inlet and outlet ports areas.

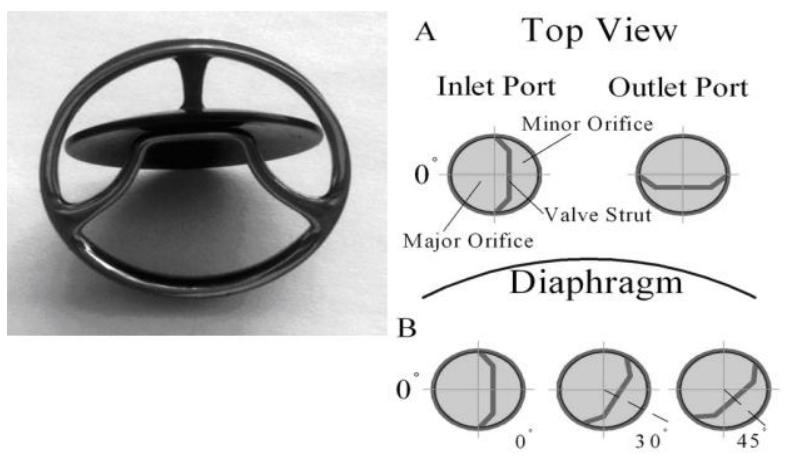

Figure 2. Tilting disk valve "MIKS" (left) and examples of the valves orientation during the modelling (A); three options of mitral valve orientation at which the modelling was realized (B) (right).

For the solution of the Navier-Stokes equations in the non-stationary statement, the hybrid (finiteelement/finite-volume) system ANSYS CFX (ANSYS, Inc., USA) [3] was used. The step of hemodynamic analysis includes creating a finite element model, the determination of the boundary conditions (Table 1) and the definition of hemodynamic parameters of the flow inside the blood chamber. This study of researches focuses on the hemodynamics inside the chamber of the $\mathrm{VAD}$ and the dynamics of the leaflets during opening and closing were not modelled, as they were shown to have only a secondary effect on the flow in the chamber [4].

Besides that, the valve closing and opening times are short compared with the duration of diastole and systole and no significant effect on the flow inside the chamber was observed [5]. The motion of the valves was not modelled. They were held fixed in their fully open or fully closed positions, depending on the phase of the cardiac cycle. During systole, the aortic valve was opened and the mitral valve was closed and during diastole, the mitral valve was opened and the aortic valve was closed. The stroke volume of the system is $30 \mathrm{cc}$. The pump rate is $75 \mathrm{bpm}$. To obtain a more accurate solution, a partition was made in accordance with the geometrical complexity of the volume. A concentrate mesh was implemented in the valves zones and in the inlet and outlet ports areas. From the diaphragm side, a change of the drive pressure took place (1), (2) and the deformation of the walls was not included. The modelling assumed a non-slip condition at the boundary.

$$
P_{\text {mem.dia }}=P_{d i a}-P_{d i a}(1+t) \sin \left(\frac{\pi}{T_{d i a}} t\right),
$$

where $t \in\left[0 ; T_{\text {dia }}\right]$.

$$
P_{\text {mem.sys }}=P_{s y s}+P_{d i a}(1+t) \sin \left(\frac{\pi}{T_{s y s}} t\right),
$$

where $t \in\left[0 ; T_{\text {sys }}\right]$.

$P_{\text {mem.dia, }} P_{\text {mem.sys }}-$ variation of the drive pressure in air chamber in diastole and systole phases; $T_{\text {dia }}, T_{\text {sys }}-$ duration of diastole and systole phases; $P_{\text {dia }}, P_{\text {sys }}-$ diastolic and systolic pressures; $t$ - current time of the phase.

The simulation evaluated the hemodynamic parameters such as velocity of the flow inside the pump chamber, Reynolds and wall shear stresses $[6,7]$.

\begin{tabular}{|c|c|}
\hline \multicolumn{2}{|l|}{ Computational model statistic: } \\
\hline Number of nodes & 298.224 \\
\hline Number of elements & $1,011,095$ \\
\hline Type of the element & tetrahedral \\
\hline Minimal length of the rib, mm & $10^{-5}$ \\
\hline Maximal length of the rib, $\mathrm{mm}$ & $7 \cdot 10^{-4}$ \\
\hline \multicolumn{2}{|c|}{ Flow parameters } \\
\hline Dynamic viscosity, $\mathrm{Pa} \cdot \mathrm{s}$ & 0.0032 \\
\hline Density, $\mathrm{kg} / \mathrm{m}^{3}$ & 1,060 \\
\hline \multicolumn{2}{|c|}{ Boundary conditions } \\
\hline Condition at the boundary & no-slip \\
\hline The model of turbulence & $\mathrm{k}-\varepsilon$ \\
\hline Pump rate, bpm & 75 \\
\hline$\beta=\mathrm{T}_{\text {sys }} / \mathrm{T}_{\text {dia }}$ & $1: 1$ \\
\hline Systolic time, s & 0.4 \\
\hline Diastolic time, $\mathrm{s}$ & 0.4 \\
\hline Outlet pressure, $\mathrm{mm} . \mathrm{Hg}$ & 100 \\
\hline Inlet pressure, $\mathrm{mm} . \mathrm{Hg}$ & 50 \\
\hline
\end{tabular}

Table 1. Model Parameters of Hemodynamic Analysis

\section{Results and Discussion}

The analysis of the modelling results that reflect the change in the flows rate, the Reynolds shear stresses and the wall shear stresses in the blood chamber of the LVAD system, allows to make preliminary conclusions about the presence of stagnation zones, which are areas of potential thrombogenesis and hemolysis. A comparison 
of the velocities fields in the diastolic phase was carried out to estimate the influence of the mitral valve orientation on the behaviour of the flow inside the LVAD system (Fig. 3). Using the tilting disc valve "MIKS" in
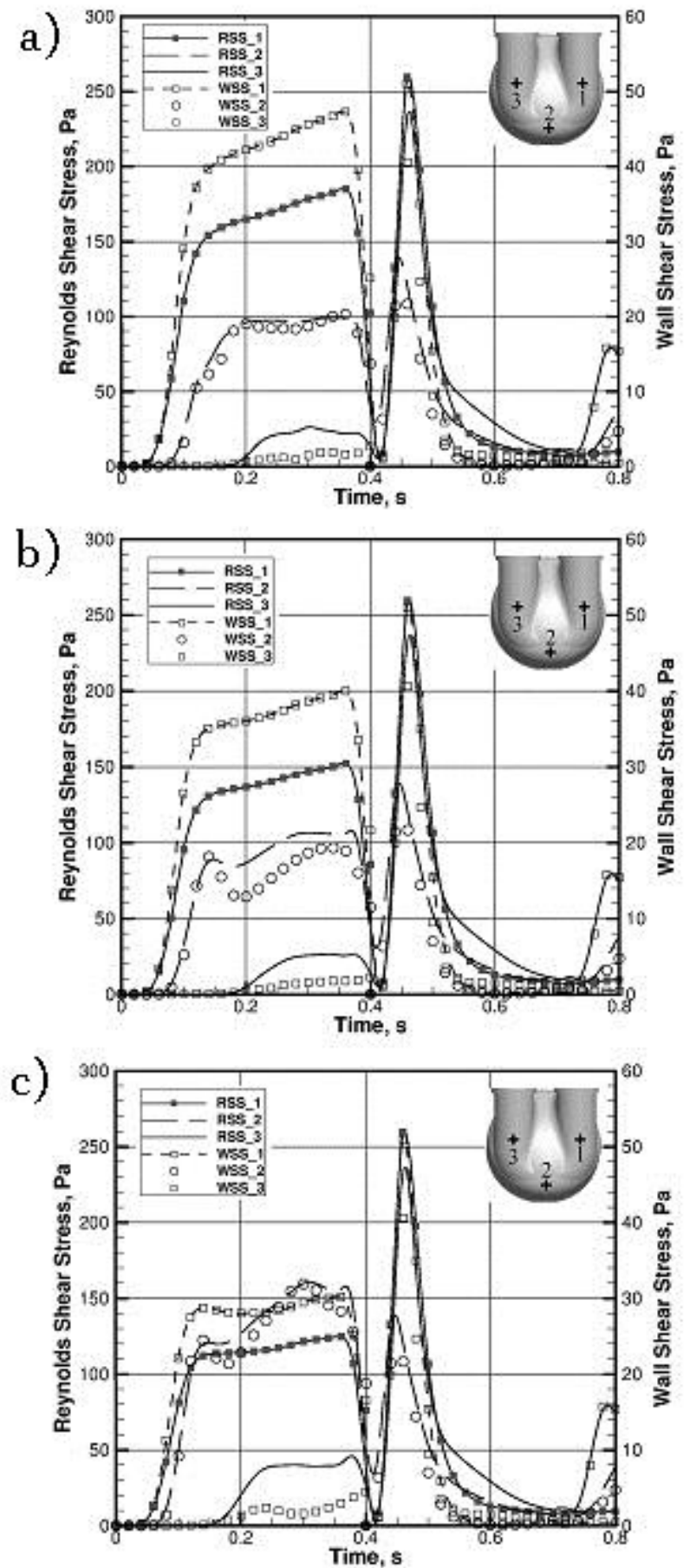

every set position, the flow is directed through the internal walls of the blood chamber and it moves in direction of the aortic valve.
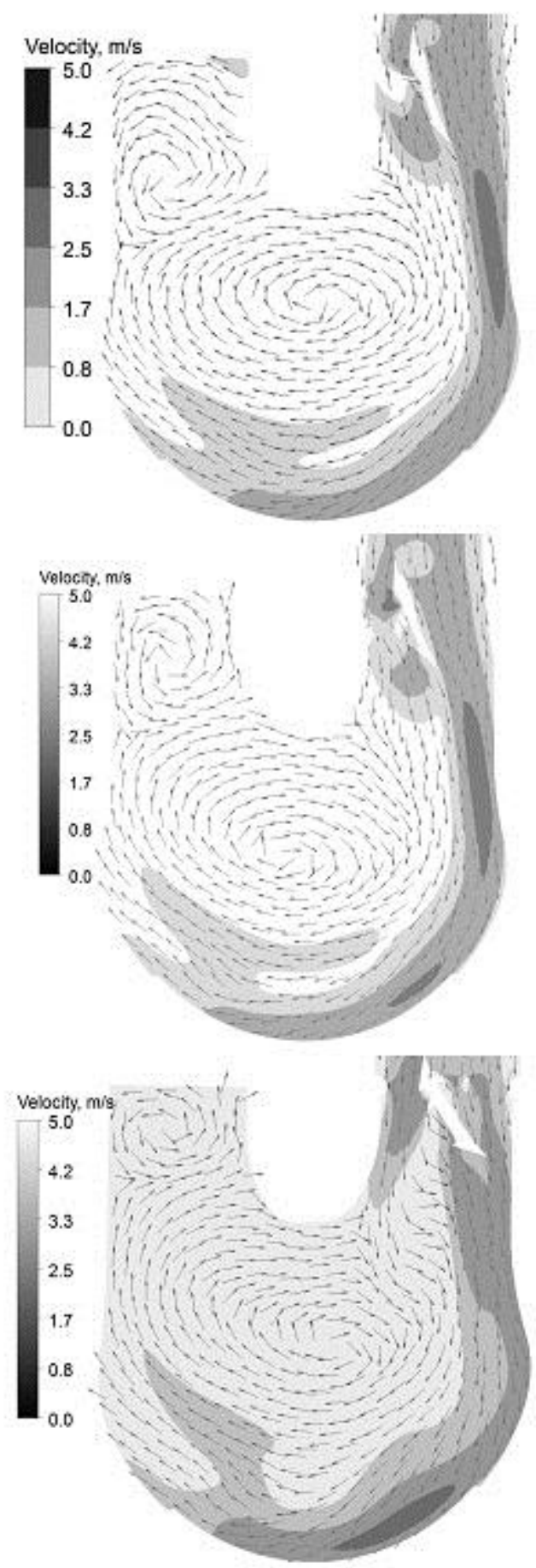

Figure 3. Cyclic variation of stresses (left) and velocity (right) magnitudes with inlet orientations of $0^{\circ}$ (a), $30^{\circ}$ (b), $45^{\circ}$ (c) and all of outlet orientations were the same at three indicated points.

The maximal flow rate is observed at its passing through the valve, but exactly through the minor orifice it decreases, falling into the blood chamber. Installing the valves at 0 and 30 degrees it is observed the union of the flows passing through the major and minor orifices, while the flow passing through the minor orifice is directed along the wall located between the inlet and outlet ports, the same for the angle of 45 degrees, and it facilitates its washing. The lack of a good internal wall washing in these areas can contribute to the formation of stagnation zones and thrombus formation. The valve installation at the angle of 45 degrees provides a better internal wall washing of the blood chamber, due to the "direction" of 
the inlet flow into the chamber. Using the valve, installed at 45 degrees, a maximal swirl flow effect of the liquid is observed inside the blood chamber in the side of the opposite branch. Figure 3 shows the change graph of the Reynolds shear stresses (RSS) and the wall shear stresses (WSS) during the cardiac cycle. Shear stresses are determined in three points: 1 - inlet port, 2 - centre of the blood chamber and 3 - outlet port for every angles of orientation of the valve. The comparison of the shear stresses values arising at different positions of the valve disk showed that the minimal stresses are caused with the valve at the angle of 45 degrees. Placing the valve at an angle of 45 degrees, the shear stresses amplitudes for the first and second points have similar values, while for angles of 0 and 30 degrees the difference between the Reynolds shear stresses and wall shear stresses is more pronounced. For both types of the valves used, the Reynolds shear stresses have bigger values than the wall shear stresses. The lower values of both types of shear stresses arising during the installation of the mitral valve at an angle of 45 degrees, facilitate a less intensive hemolysis of the erythrocytes and improve the hemodynamic indicator of the LVAD system.

Application of the rapid prototyping technologies allowed to obtain as single elements of the device as the complete assembly of the product (Fig. 4) from the materials, of which the properties are similar to the properties of materials [8] planed to apply them during the manufacture of LVAD systems for in-vivo tests. The prototype's development allowed to evaluate the appearance of the designed device, its implantability and also to carry out the hydrodynamic test to evaluate its efficiency without manufacturing items with additional accessories, and hence to obtain a considerable saving in time.

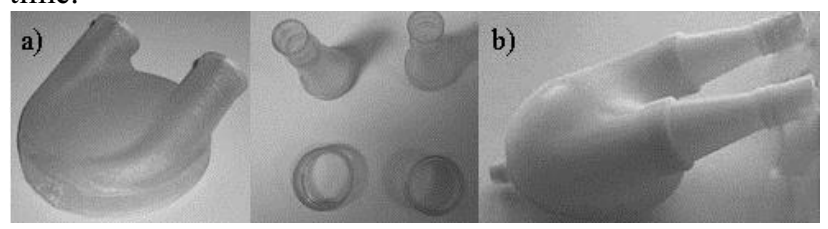

Figure 4. The pneumatic LVAD components manufactured using rapid prototyping technologies: housing of the blood pump and connectors (a), LVAD assembly (b).

However, the application of these technologies for manufacturing blood pumps is not appropriate due to the materials used in prototyping, which are not approved by the Ministry of Health of the Russian Federation to apply it in medical equipment.
In this work it was examined the possibility of application of $\mathrm{CAD} / \mathrm{CFD} / \mathrm{RP}$ technologies during the design of LVAD systems. The main focus is on the evaluation of the influence of the position of the mitral valve in hydrodynamic processes occurring inside the blood chamber during the operation of the LVAD system. The obtained results showed that the use of the tilting disk valve "MIKS" commercially available in Russia oriented at a 45 degrees angle provides a good flow internal wall washing of the blood chamber of the LVAD system. A good wall washing and swirl flow reduce the thrombosis risk during the operation of the LVAD system. The absence of stagnation zones, recirculation flow zones, allow us to talk about an acceptable design of the blood chamber. The application of CAD/CFD/RP-technologies allows accelerating the design process of LVAD systems. The manufactured components of the LVAD system are completely operability and give the possibility to estimate the critical hemodynamic and functional parameters of LVAD system.

\section{Acknowledgment}

Financial support: This research was supported by the Presidential Program of Support for Young Candidates of Science (project no. MK-5860.2015.8).

\section{References}

1. L. V. Belyaev, A. B. Ivanchenko, A. V. Zhdanov, V. V.Morozov, Biomed. Eng., 49, 1, 24-28 (2015).

2. LLC "Roskardioinvest". 2016. The mechanical "MIKS" heart valves. [ONLINE] Available at: http://roscardioinvest.ru/eng/index.php?id_subpart=1 0 .

3. ANSYS, Inc. ANSYS CFX Technical Brief, 2015. Release 14.0. Available at: http://www.ansys.com/staticassets/ansys/staticassets /resourcelibrary/techbrief/cfx-numerics.pdf.

4. M. Rosenfeld, I. Avrahami, S. Einav, J. Biomech. Eng., 124, 21-29 (2002).

5. I. Avrahami, M. Rosenfeld, S. Raz, S. Einav, Artif. Organs. 30, 7, 529-538 (2006).

6. K. Okamoto, T. Hashimoto, Y. Mitamura, J. Artif. Organs, 6, 3, $162-167$ (2003).

7. E. Okamoto, S. Fukuoka, E. Iwasawa, Y. Mitamura J. Artif. Organs, 4, 205-213 (2001).

8. V.V. Morozov, A. V. Zhdanov, Mechatronics, automation, control, 11, 30 - 31, (2008), (in Russian).

\section{Conclusions}

